\title{
La comunicación en las empresas de servicio
}

\author{
Luis Nieri \\ José Ferreccio
}

El nuevo rol que se está otorgando a las comunicaciones en las empresas de servicios es un tema que va cobrando interés en los últimos tiempos. Por ello, Contratexto ha considerado de utilidad recoger en las páginas que siguen la conversación que sostuvieran Adriana Castillo y Mireya Guerrero, egresadas de la Facultad de Ciencias de la Comunicación de la Universidad de Lima con Luis Nieri, encargado de la Secretaría de Relaciones Institucionales del Banco de Crédito del Perú y José Ferreccio, gerente adjunto de Planeamiento y Organización del Banco Continental.

Un primer eje temático estuvo encaminado a conocer los nuevos espacios que están ocupando los responsables de las acciones de comunicación en las empresas de servicios en banca y la adaptación y procesos de socialización de los nuevos trabajadores.

Un segundo bloque de preguntas tuvo relación con el rol que se asigna en estas empresas a las comunicaciones en la generación de la cultura empresarial y el papel que desempeñan en la implementación y adecuación de los cambios.

¿Qué rol se le asigna en sus empresas a las comunicaciones cuando se trata de paliar los efectos de los procesos de adaptación del personal?
Luis Nieri. Si bien no podemos decir que en el Banco existe un catecismo o que tenemos un evangelio, tratamos de que en los tres primeros meses de labor se absorban los valores propios de la institución; $\mathrm{y}$, en este aspecto, las comunicaciones desempeñan un rol de mucha importancia. Además, el área de Recursos Humanos del Banco integra a los padres de familia del personal nuevo, generando todo un sentimiento de pertenencia.

No obstante que no contamos actualmente con un video institucional, les entregamos libros y folletos que hacen su incorporación más fácil, pero a su vez le brindan la satisfacción de pertenecer a una institución importante.

José Ferreccio. Los procesos de adaptación al Banco están cuidadosamente planeados por el Área de Recursos Humanos. Se desea que el trabajador sienta que es bienvenido y que el Banco quiere darle todas las facilidades para que sus funciones las desempeñe con satisfacción y con eficacia. Primero, se hacen actividades a nivel local (el área o sección donde se va a desempeñar), luego tenemos el video institucional y los documentos que les permiten conocer los objetivos de la institución y lo que se espera de cada uno; finalmente, se programan diversas etapas de training, de acuerdo con el puesto que tiene que desempeñar. 
L.N. En esta etapa, la regularidad y prontitud de las comunicaciones es vital. El nuevo trabajador debe sentir que se preocupan por él y que el Banco está pendiente de sus necesidades. Se busca, además, darle seguridad y que esta satisfacción y seguridad se vea reflejada en el tratamiento con los públicos con los que tiene que interactuar. En todo momento debe ser consciente de que representa al banco.

J.F. Dependiendo del nivel de la persona que incorporamos, los procesos de comunicación son más constantes. Es importante que sepa todo lo que hay que saber sobre el Banco y sobre las funciones que va a desempeñar. Ello repercute en su satisfacción y en el trato al público.

Conscientes de la importancia de esta fase, el Banco usa todos los medios de comunicación disponibles para estar en contacto con los recién ingresantes. Además promovemos, desde el inicio, una actitud de participación.

¿Cómo se transmiten los valores importantes de sus empresas a los nuevos trabajadores y qué papel desempeñan las comunicaciones?

L.N. En todo proceso de adaptación es preciso utilizar una estrategia racional para lograr una buena incorporación. Todo proceso en el que las comunicaciones permanentes son importantes, se inicia por lo genérico: la historia del Banco, los objetivos de la empresa, la familiarización con los procedimientos y con sus funciones. Luego se inculca la necesidad de mantener el liderazgo del Banco, de brindar el mejor servicio posible e incluso se motiva a los trabajadores a reducir costos, pero a la vez a brindar un buen servicio.

En todos estos momentos el personal cuenta con gente de Recursos Humanos que está a su lado para resolver las dudas y hacer las aclaraciones necesarias, pero más importante es la comunicación directa con las personas que están cerca de él y por eso se fomenta desde el inicio mantener una comunicación abierta con los que son sus compañeros de trabajo. Este aspecto de la comunicación interpersonal tiene bastante importancia para nosotros.

J.F. Somos muy cuidadosos con la implementación de estos procesos iniciales. Recursos Humanos tiene mucha experiencia en esto y planifica las actividades con meticulosidad. A los nuevos se les da suficiente material para lectura. Este material ha sido cuidadosamente elaborado y los comunicadores juegan un papel importante tanto en su diagramación y presentación como en el contenido. Se entrega un documento síntesis de la organización del Banco, de los objetivos y los principios básicos del Banco, su misión y su visión, así como el reglamento interno de trabajo. Luego sigue un curso de inducción de seis a siete dias, donde se pasan videos; pero fundamentalmente se resuelven preguntas, dudas y se fomenta un espíritu de participación y aporte para el mejoramiento del Banco. Las evaluaciones de estas actividades son procesadas con mucho detenimiento para mejorarlas.

L.N. El Banco no limita estos eventos de transmisión de valores al personal nuevo, sino que constantemente todos nos estamos actualizando y capacitando. Tratamos de que las comunicaciones no sean rígidas ni programadas, buscamos que sean naturales e inculcamos la necesidad de una comunicación abierta, donde la relación personal es básica. En una institución con tanto personal tratamos de no ser muy formales, ni rígidos en los procesos de comunicación. A todos se inculca los valores señalados, pero a través de una comunicación bastante fluida. 
J.F. Para este tipo de actividades, el Banco cuenta con asesoría externa y los resultados son bastante buenos. Sin embargo, lo esencial, que es la transmisión de valores y la identificación con el banco, es de manejo interno. Se busca que desde el inicio el trabajador participe en la marcha de su institución y por ello tratamos de incentivar los mecanismos que les posibilite aportar a la mejor marcha del Banco. Ello les da una seguridad de pertenecer al Banco, pero a su vez se refleja en el trato con el público.

Por otra parte, los antiguos trabajadores, con asesoría externa, están recibiendo cursos sobre la actitud frente al cambio, especialmente necesarios en estos momentos de transición, donde incluso se les prepara para que realicen otras actividades fuera del Banco. Lo importante para la institución es que ellos sientan que el Banco está a su lado y que esto les permita un trabajo eficiente. Especialmente nos preocupamos por los que tienen un contacto directo con el público, pues queremos construir una imagen de servicio. Acá los comunicadores juegan un rol de primer nivel, pues estos procesos son dificiles, aparecen las naturales resistencias. Por eso formamos equipos multidisciplinarios que tienen en cuenta todos estos aspectos.

¿Cómo se percibe en sus instituciones la formación de la cultura empresarial y cómo se ve el rol de las comunicaciones en este campo?

L.N. Todas las instituciones, sin excepción, conforman una cultura organizacional. En nuestro caso ha llevado tiempo hacerla y la intención es mantenerla, modificando aquellos aspectos que se vuelven obsoletos. Para ello es importante generar mecanismos que nos permitan re- coger las preocupaciones de todos y cada uno de los trabajadores. Por ello, los procesos de evaluación deben estar en manos de profesionales responsables de evaluar estos nuevos elementos culturales y saber darlos a conocer a los demás. Las comunicaciones planificadas juegan un papel importante.

Estamos dejando las calificaciones subjetivas sobre aspectos tales como identificación, cooperación, etc., y con el apoyo de diversos especialistas, incluyendo comunicadores, estamos diseñando instrumentos cuantificables para conocer mejor la conducta de las personas. Así tendremos una idea más clara de nuestro personal y de la cultura organizacional presente en el Banco. Luego, con la gente de comunicaciones y de psicología se implementan actividades de cambio y de modificación de la cultura empresarial.

J.F. En el Banco hemos creado una serie de lemas: "El Banco amigo", "Todos somos amigos", "Amigos para toda la vida", etc., basados en estudios de opinión, en función tanto del público externo como del público interno; con ellos tratamos de resaltar parte de la cultura institucional. "El Banco es tu amigo", tanto para los que laboran dentro de él como para el público externo. Esta filosofia lleva a brindar una excelente calidad en la atención y a generar confianza en el público que hace uso de nuestros servicios.

La cultura en instituciones de servicios, especialmente las bancarias, debe responder tanto a las necesidades de los que laboran en la institución, como a las del público que viene al Banco. El estudio de estas dos vertientes de elementos culturales permitirá una mejor compenetración con la calidad del servicio. Tanto en la fase de estudios de estos valores como en el proceso de consolidación, las comunica- 
ciones tienen un papel de primer orden. Conocer lo que existe y saber cómo presentarlo de manera que genere poca resistencia, es tarea de los comunicadores y de otros profesionales de nuestro Banco.

L.N. Es preciso enfatizar que en el Banco se han generado mecanismos que permiten una participación directa de los funcionarios y del personal con responsabilidad en la marcha de los procesos importantes del Banco. Igualmente, se han implementado un conjunto de procedimientos para recibir sugerencias de los trabajadores y del público; éstas son evaluadas y estudiadas con detenimiento. Las reuniones permanentes de las gerencias con los directivos del Banco nos permiten hacer un seguimiento cercano de todos los procesos de cambio y de su impacto en el Banco. Para el diseño de este proceso de recolección de opiniones y sugerencias se cuenta con los profesionales de las ciencias de la comunicación, lo mismo que cuando realizamos campañas internas.

J.F. Podemos señalar que el sistema de comunicación diseñado por el Banco y enseñado a los trabajadores es el que nos permite tener un notable éxito en la evaluación de los procesos de cambio. Mucha gente del Banco participa en diversos tipos de reuniones en la institución y ello les permite, por un lado, estar bastante bien informados, $y$, por otra, aportar en la marcha del Banco. Igualmente, en cuanto a la capacitación tratamos de mantener a nuestro personal actualizado y con mentalidad de cambio y modernidad. La revista Top nos ha considerado como una de las mejores escuelas bancarias. Estos méritos son el esfuerzo de varias personas, pero sobre todo de los equipos que se dedican al estudio de los mecanismos de comunicación en nuestra institución. 